\title{
LOCAL WELL-POSEDNESS FOR THE HALL-MHD SYSTEM IN OPTIMAL SOBOLEV SPACES
}

\author{
MIMI DAI
}

\begin{abstract}
We show that the viscous resistive magneto-hydrodynamics system with Hall effect is locally well-posed in $H^{s}\left(\mathbb{R}^{n}\right) \times H^{s+1-\varepsilon}\left(\mathbb{R}^{n}\right)$ with $s>\frac{n}{2}-1$ and any small enough $\varepsilon>0$ such that $s+1-\varepsilon>\frac{n}{2}$. This space is to date the largest local well-posedness space in the class of Sobolev spaces for the system. It is also optimal according to the predominant scalings of the two equations in the system.
\end{abstract}

KEY WORDS: Magneto-hydrodynamics; Hall effect; local well-posedness; scaling structure.

CLASSIFICATION CODE: 76D03, 35Q35.

\section{INTRODUCTION}

Considered in this treatise is the three dimensional incompressible viscous resistive Hall-magneto-hydrodynamics (Hall-MHD) system:

$$
\begin{array}{r}
u_{t}+u \cdot \nabla u-b \cdot \nabla b+\nabla p-\nu \Delta u=0, \\
b_{t}+u \cdot \nabla b-b \cdot \nabla u+\eta \nabla \times((\nabla \times b) \times b)-\mu \Delta b=0, \\
\nabla \cdot u=0,
\end{array}
$$

accompanied with the initial conditions

$$
u(x, 0)=u_{0}(x), \quad b(x, 0)=b_{0}(x), \quad \nabla \cdot u_{0}=\nabla \cdot b_{0}=0,
$$

for $x \in \mathbb{R}^{3}$ and $t \geq 0$. In the system, $u$ represents the fluid velocity, $p$ is the fluid pressure and $b$ stands for the magnetic field. The parameters $\nu, \mu$ and $\eta$ denote the fluid viscosity, resistivity (electrical diffusivity) and the Hall effect coefficient, respectively. It is important to observe that, if $\nabla \cdot b_{0}=0$, the divergence free condition for $b$ is propagated by the second equation of (1.1), see 4. The Hall term $\nabla \times((\nabla \times b) \times b)$ distinguishes (1.1) from the usual MHD system (system (1.1) with $\eta=0)$. In contrast to the latter one, the Hall-MHD model is more advantageous due to the fact that it can capture the essential characteristics of the magneto-hydrodynamics with strong magnetic reconnection where the Hall effect plays a significant role. Magnetic reconnection is a fundamental dynamical process in highly conductive plasmas in astrophysics, allowing for explosive and efficient magnetic to kinetic energy conversion. For a more comprehensive physical background of the magnetic reconnection phenomena and the Hall-MHD model, we refer the readers to [11, 14, 16] and references therein.

Despite its increasing popularity among the astrophysicists community, the mathematical understanding of the Hall-MHD model is very limited. Conceptually, we can have a peek about the barriers from various perspectives. First, the Hall term launches new physics into the system at small length scales and hence intrinsically 
challenging into the mathematical analysis. Second, it is well-known that the main obstacle to understand the turbulent flows governed by the Navier-Stokes equation (NSE) relies on the nonlinearity such as $(u \cdot \nabla) u$. One can imagine that system (1.1) is more intricate than the NSE, for the former one contains the NSE and a magnetic field equation with the Hall term which appears more singular than $(u \cdot \nabla) u$. Third, the natural scaling structure is a strong motivation in the study of both the NSE and the MHD system, who share the same scaling. However, the Hall term destroys such natural scaling. Into more details, for the MHD system, if $(u(x, t), p(x, t), b(x, t))$ solves (1.1) with $\eta=0$ with the initial data $\left(u_{0}(x), b_{0}(x)\right)$, then the triplet $\left(u_{\lambda}(x, t), p_{\lambda}(x, t), b_{\lambda}(x, t)\right)$ defined by

$$
u_{\lambda}(x, t)=\lambda u\left(\lambda x, \lambda^{2} t\right), \quad p_{\lambda}(x, t)=\lambda^{2} p\left(\lambda x, \lambda^{2} t\right), \quad b_{\lambda}(x, t)=\lambda b\left(\lambda x, \lambda^{2} t\right)
$$

solves the same system with the data

$$
u_{0 \lambda}(x, t)=\lambda u_{0}(\lambda x), \quad b_{0 \lambda}(x, t)=\lambda b_{0}(\lambda x) .
$$

The scaling (1.3) no longer holds for system (1.1) with $\eta>0$. On the other hand, we can extract the "Hall equation"

$$
b_{t}+\nabla \times((\nabla \times b) \times b)=\Delta b
$$

which has the scaling

$$
b_{\lambda}(x, t)=b\left(\lambda x, \lambda^{2} t\right) .
$$

Since the Hall term is the most singular nonlinearity in system (1.1), it suggests that the predominant scaling for (1.1) could be

$$
u_{\lambda}(x, t)=\lambda u\left(\lambda x, \lambda^{2} t\right), \quad p_{\lambda}(x, t)=\lambda^{2} p\left(\lambda x, \lambda^{2} t\right), \quad b_{\lambda}(x, t)=b\left(\lambda x, \lambda^{2} t\right) .
$$

In fact, based on scaling (1.5), we obtained a regularity criterion for (1.1) in three dimension which improves various criteria in the literature, see 9].

In this paper our interest is to find the largest possible (optimal) Sobolev space where system (1.1) is locally well-posed. On this topic, it was first shown in [6] that system (1.1) in three dimension is locally well-posed in $H^{s}\left(\mathbb{R}^{3}\right) \times H^{s}\left(\mathbb{R}^{3}\right)$ with $s>\frac{5}{2}$. By taking (1.4) as the dominant scaling, in [8], we obtained the local well-posedness of (1.1) in $H^{s}\left(\mathbb{R}^{n}\right) \times H^{s}\left(\mathbb{R}^{n}\right)$ with $s>\frac{n}{2}$. Even though the result of [8] improves that of [6], it seems that there is still room to have improvement, for the reason that the NSE is known to be locally well-posed in $H^{s}\left(\mathbb{R}^{n}\right)$ with $s>\frac{n}{2}-1$. In fact, motivated by scaling (1.5), one expects that system (1.1) may be locally well-posed in $H^{s}\left(\mathbb{R}^{n}\right) \times H^{s+1}\left(\mathbb{R}^{n}\right)$ with $s>\frac{n}{2}-1$. In order to justify the conjecture, we need to treat the energy estimates for $u$ and $b$ separately, namely, $u$ in $H^{s}$ and $b$ in $H^{s+1}$. In this situation, we encounter the difficulty that no cancelation can be employed to deal with the two terms $b \cdot \nabla b$ and $b \cdot \nabla u$. To overcome this barrier, it comes to our mind that we need to optimize the estimates of the flux contributed from the two terms by fully employing the diffusion of both the $u$ and the $b$. Techniques based on the paradifferential calculus enables us to operate such optimizations. Surprisingly, it turns out that the local well-posedness space we can obtain is slightly larger than the conjectured one. In deed, we prove the main result below.

Theorem 1.1. Let $\left(u_{0}, b_{0}\right) \in H^{s}\left(\mathbb{R}^{n}\right) \times H^{s+1-\varepsilon}\left(\mathbb{R}^{n}\right)$ with $s>\frac{n}{2}-1$ and any small enough $\varepsilon>0$ such that $s+1-\varepsilon>\frac{n}{2}$. Assume $\nabla \cdot u_{0}=\nabla \cdot b_{0}=0$. There exists a 
time $T=T\left(\nu, \mu,\left\|u_{0}\right\|_{H^{s}},\left\|b_{0}\right\|_{H^{s+1-\varepsilon}}\right)>0$ and a unique solution $(u, b)$ of (1.1) on $[0, T]$ such that

$$
(u, b) \in C\left([0, T) ; H^{s}\left(\mathbb{R}^{n}\right)\right) \times C\left([0, T) ; H^{s+1-\varepsilon}\left(\mathbb{R}^{n}\right)\right) .
$$

Regarding the result, the fact that $b$ needs to be in a space with higher regularity is determined by the Hall term. Predicted by the scaling (1.4) of the "Hall equation", the optimal Sobolev space of well-posedness for $b$ would be $H^{s+1}\left(\mathbb{R}^{n}\right)$ with $s>$ $\frac{n}{2}-1$. However, as stated in Theorem 1.1 the obtained well-posdness space for $b$ is $H^{s+1-\varepsilon}\left(\mathbb{R}^{n}\right)$ for any small $\varepsilon>0$. It may be explained by getting a closer look at the term $b \cdot \nabla u$. While estimating $\|b \cdot \nabla u\|_{H^{r}}$ by applying both diffusions of $u$ and $b$, it happens that we need to take $r$ slightly smaller than $s+1$.

\section{Preliminaries}

2.1. Notation. In order to avoid confusion, we specify a few notations. We denote by $A \lesssim B$ an estimate of the form $A \leq C B$ with some absolute constant $C$, and by $A \sim B$ an estimate of the form $C_{1} B \leq A \leq C_{2} B$ with absolute constants $C_{1}, C_{2}$. For simplification, it is understood that $\|\cdot\|_{p}=\|\cdot\|_{L^{p}}$.

2.2. Littlewood-Paley decomposition. As in our previous articles on the local well-posedness of magneto-hydrodynamics systems, the main tool is paradifferential calculus. To be self-contained, we recall the Littlewood-Paley decomposition theory briefly, even though it appears in our earlier work on related topics. For a more detailed description on this theory we refer the readers to 2 and [12].

Let $\mathcal{F}$ and $\mathcal{F}^{-1}$ denote the Fourier transform and inverse Fourier transform, respectively. Define $\lambda_{q}=2^{q}$ for integers $q$. A nonnegative radial function $\chi \in$ $C_{0}^{\infty}\left(\mathbb{R}^{n}\right)$ is chosen such that

$$
\chi(\xi)= \begin{cases}1, & \text { for }|\xi| \leq \frac{3}{4} \\ 0, & \text { for }|\xi| \geq 1\end{cases}
$$

Let

$$
\varphi(\xi)=\chi\left(\frac{\xi}{2}\right)-\chi(\xi)
$$

and

$$
\varphi_{q}(\xi)=\left\{\begin{array}{l}
\varphi\left(\lambda_{q}^{-1} \xi\right) \quad \text { for } q \geq 0, \\
\chi(\xi) \quad \text { for } q=-1
\end{array}\right.
$$

For a tempered distribution vector field $u$ we define the Littlewood-Paley projection

$$
\begin{aligned}
& h=\mathcal{F}^{-1} \varphi, \quad \tilde{h}=\mathcal{F}^{-1} \chi, \\
& u_{q}:=\Delta_{q} u=\mathcal{F}^{-1}\left(\varphi\left(\lambda_{q}^{-1} \xi\right) \mathcal{F} u\right)=\lambda_{q}^{n} \int h\left(\lambda_{q} y\right) u(x-y) d y, \quad \text { for } q \geq 0, \\
& u_{-1}=\mathcal{F}^{-1}(\chi(\xi) \mathcal{F} u)=\int \tilde{h}(y) u(x-y) d y .
\end{aligned}
$$

By the Littlewood-Paley theory, the identity

$$
u=\sum_{q=-1}^{\infty} u_{q}
$$


holds in the distributional sense. For brevity, we agree with the notations

$$
u_{\leq Q}=\sum_{q=-1}^{Q} u_{q}, \quad u_{(Q, N]}=\sum_{p=Q+1}^{N} u_{p}, \quad \tilde{u}_{q}=\sum_{|p-q| \leq 1} u_{p} .
$$

Definition 2.1. A tempered distribution $u$ belongs to the Besov space $B_{p, \infty}^{s}$ if and only if

$$
\|u\|_{B_{p, \infty}^{s}}=\sup _{q \geq-1} \lambda_{q}^{s}\left\|u_{q}\right\|_{p}<\infty .
$$

We can identify the Sobolev space $H^{s}$ by the Besov space $B_{2,2}^{s}$, i.e.

$$
\|u\|_{H^{s}} \sim\left(\sum_{q=-1}^{\infty} \lambda_{q}^{2 s}\left\|u_{q}\right\|_{2}^{2}\right)^{1 / 2}
$$

for each $u \in H^{s}$ and $s \in \mathbb{R}$.

Lemma 2.2. (Bernstein's inequality. See [13.) Let $n$ be the space dimension and $r \geq s \geq 1$. Then for all tempered distributions $u$, we have

$$
\left\|u_{q}\right\|_{r} \lesssim \lambda_{q}^{n\left(\frac{1}{s}-\frac{1}{r}\right)}\left\|u_{q}\right\|_{s} .
$$

2.3. Bony's paraproduct and commutator. Bony's paraproduct formula

$$
\begin{aligned}
\Delta_{q}(u \cdot \nabla v)= & \sum_{|q-p| \leq 2} \Delta_{q}\left(u_{\leq p-2} \cdot \nabla v_{p}\right)+\sum_{|q-p| \leq 2} \Delta_{q}\left(u_{p} \cdot \nabla v_{\leq p-2}\right) \\
& +\sum_{p \geq q-2} \Delta_{q}\left(\tilde{u}_{p} \cdot \nabla v_{p}\right)
\end{aligned}
$$

will be used constantly to decompose the nonlinear terms in energy estimate. We will also use the notation of the commutator

$$
\left[\Delta_{q}, u_{\leq p-2} \cdot \nabla\right] v_{p}:=\Delta_{q}\left(u_{\leq p-2} \cdot \nabla v_{p}\right)-u_{\leq p-2} \cdot \nabla \Delta_{q} v_{p} .
$$

Lemma 2.3. The commutator satisfies the following estimate, for any $1<r<\infty$

$$
\left\|\left[\Delta_{q}, u_{\leq p-2} \cdot \nabla\right] v_{p}\right\|_{r} \lesssim\left\|\nabla u_{\leq p-2}\right\|_{\infty}\left\|v_{p}\right\|_{r} .
$$

2.4. Auxiliary estimates. To handle the Hall term $\nabla \times((\nabla \times b) \times b)$, more preparation is needed. We first introduce two more commutators and their estimates. We define that, for vector valued functions $F$ and $G$,

$$
\begin{gathered}
{\left[\Delta_{q}, F \times \nabla \times\right] G=\Delta_{q}(F \times(\nabla \times G))-F \times\left(\nabla \times G_{q}\right),} \\
{\left[\Delta_{q}, \nabla \times F \times\right] G=\Delta_{q}(\nabla \times F \times G)-\nabla \times F \times G_{q} .}
\end{gathered}
$$

In principle, the commutators will be used to reveal certain cancellation; and to shift derivative from high modes to low modes. It was shown in [9] they satisfy the following estimates.

Lemma 2.4. Let $F$ and $G$ be vector valued functions. Assume $\nabla \cdot F=0$ and $F$, $G$ vanish at large $|x| \in \mathbb{R}^{3}$. For any $1<r<\infty$, we have

$$
\begin{gathered}
\left\|\left[\Delta_{q}, F \times \nabla \times\right] G\right\|_{r} \lesssim\|\nabla F\|_{\infty}\|G\|_{r} ; \\
\left\|\left[\Delta_{q}, \nabla \times F \times\right] G\right\|_{r} \lesssim\|\nabla F\|_{\infty}\|G\|_{r} .
\end{gathered}
$$


Lemma 2.5. Let $F, G$ and $H$ be vector valued functions. Assume $F, G$ and $H$ vanish at large $|x| \in \mathbb{R}^{3}$. For any $1<r_{1}, r_{2}<\infty$ with $\frac{1}{r_{1}}+\frac{1}{r_{2}}=1$, we have

$$
\left|\int_{\mathbb{R}^{3}}\left[\Delta_{q}, \nabla \times F \times\right] G \cdot \nabla \times H d x\right| \lesssim\left\|\nabla^{2} F\right\|_{\infty}\|G\|_{r_{1}}\|H\|_{r_{2}} .
$$

\section{A pRIORI estimate}

In this section, we establish a priori estimate for smooth solutions in $H^{s}\left(\mathbb{R}^{n}\right) \times$ $H^{r}\left(\mathbb{R}^{n}\right)$ with appropriate index $s$ and $r$. Such estimate is the most crucial ingredient in the argument of local well-posedness, which is rather standard for dissipative equations, see [15]. Thus we only present the following theorem and its proof.

Theorem 3.1. Let $\left(u_{0}, b_{0}\right) \in H^{s}\left(\mathbb{R}^{n}\right) \times H^{r}\left(\mathbb{R}^{n}\right)$ with $s>\frac{n}{2}-1$ and $\frac{n}{2}<r \leq s+1-\varepsilon$ for small enough $\varepsilon>0$. There exists a time $T=T\left(\nu, \mu,\left\|u_{0}\right\|_{H^{s}},\left\|b_{0}\right\|_{H^{r}}\right)>0$ such that the Hall-MHD system (1.1) has a solution $(u, b)$ satisfying

$$
\begin{aligned}
& u \in L^{\infty}\left(0, T ; H^{s}\left(\mathbb{R}^{n}\right)\right) \cap L^{2}\left(0, T ; H^{s+1}\left(\mathbb{R}^{n}\right)\right), \\
& b \in L^{\infty}\left(0, T ; H^{r}\left(\mathbb{R}^{n}\right)\right) \cap L^{2}\left(0, T ; H^{r+1}\left(\mathbb{R}^{n}\right)\right) .
\end{aligned}
$$

The proof involves certain amount of computations and estimates which will be divided into several lemmas, each carrying an estimate for a flux term. To start, multiplying the first equation of (1.1) by $\lambda_{q}^{2 s} \Delta_{q} u_{q}$ and the second one by $\lambda_{q}^{2 r} \Delta_{q} b_{q}$, and adding up for all $q \geq-1$, we obtain

$$
\begin{gathered}
\frac{1}{2} \frac{d}{d t} \sum_{q \geq-1} \lambda_{q}^{2 s}\left\|u_{q}\right\|_{2}^{2}+\nu \sum_{q \geq-1} \lambda_{q}^{2 s+2}\left\|u_{q}\right\|_{2}^{2} \leq-I_{1}-I_{2}, \\
\frac{1}{2} \frac{d}{d t} \sum_{q \geq-1} \lambda_{q}^{2 r}\left\|b_{q}\right\|_{2}^{2}+\mu \sum_{q \geq-1} \lambda_{q}^{2 r+2}\left\|b_{q}\right\|_{2}^{2} \leq-I_{3}-I_{4}-I_{5},
\end{gathered}
$$

with

$$
\begin{aligned}
& I_{1}=\sum_{q \geq-1} \lambda_{q}^{2 s} \int_{\mathbb{R}^{3}} \Delta_{q}(u \cdot \nabla u) \cdot u_{q} d x, \quad I_{2}=-\sum_{q \geq-1} \lambda_{q}^{2 s} \int_{\mathbb{R}^{3}} \Delta_{q}(b \cdot \nabla b) \cdot u_{q} d x, \\
& I_{3}=\sum_{q \geq-1} \lambda_{q}^{2 r} \int_{\mathbb{R}^{3}} \Delta_{q}(u \cdot \nabla b) \cdot b_{q} d x, \quad I_{4}=-\sum_{q \geq-1} \lambda_{q}^{2 r} \int_{\mathbb{R}^{3}} \Delta_{q}(b \cdot \nabla u) \cdot b_{q} d x, \\
& I_{5}=-\sum_{q \geq-1} \lambda_{q}^{2 r} \int_{\mathbb{R}^{3}} \Delta_{q}((\nabla \times b) \times b) \cdot \nabla \times b_{q} d x .
\end{aligned}
$$

To fully exploit cancelations in the flux terms $I_{1}, I_{3}$ and $I_{5}$, we will apply commutator estimates along with Bony's paraproduct and some fundamental inequalities. While $r \neq s$, there is no cancelation in $I_{2}+I_{4}$, and hence $I_{2}$ and $I_{4}$ will be treated in slightly different ways.

Lemma 3.2. Let $s>\frac{n}{2}-1$. We have that, for some absolute constants $\gamma_{1}, \gamma_{2}>0$,

$$
\left|I_{1}\right| \leq \frac{\nu}{8} \sum_{q \geq-1} \lambda_{q}^{2 s+2}\left\|u_{q}\right\|_{2}^{2}+C_{\nu}\|u\|_{H^{s}}^{2+\gamma_{1}}+C_{\nu}\|u\|_{H^{s}}^{2+\gamma_{2}} .
$$


Proof: Using Bony's paraproduct (2.7) followed by the commutator notation (2.8), $I_{1}$ is decomposed as

$$
\begin{aligned}
I_{1}= & \sum_{q \geq-1} \sum_{|q-p| \leq 2} \lambda_{q}^{2 s} \int_{\mathbb{R}^{3}} \Delta_{q}\left(u_{\leq p-2} \cdot \nabla u_{p}\right) u_{q} d x \\
& +\sum_{q \geq-1} \sum_{|q-p| \leq 2} \lambda_{q}^{2 s} \int_{\mathbb{R}^{3}} \Delta_{q}\left(u_{p} \cdot \nabla u_{\leq p-2}\right) u_{q} d x \\
& +\sum_{q \geq-1} \sum_{p \geq q-2} \lambda_{q}^{2 s} \int_{\mathbb{R}^{3}} \Delta_{q}\left(u_{p} \cdot \nabla \tilde{u}_{p}\right) u_{q} d x \\
= & I_{11}+I_{12}+I_{13},
\end{aligned}
$$

with

$$
\begin{aligned}
I_{11}= & \sum_{q \geq-1} \sum_{|q-p| \leq 2} \lambda_{q}^{2 s} \int_{\mathbb{R}^{3}}\left[\Delta_{q}, u_{\leq p-2} \cdot \nabla\right] u_{p} u_{q} d x \\
& +\sum_{q \geq-1} \sum_{|q-p| \leq 2} \lambda_{q}^{2 s} \int_{\mathbb{R}^{3}} u_{\leq q-2} \cdot \nabla \Delta_{q} u_{p} u_{q} d x \\
& +\sum_{q \geq-1} \sum_{|q-p| \leq 2} \lambda_{q}^{2 s} \int_{\mathbb{R}^{3}}\left(u_{\leq p-2}-u_{\leq q-2}\right) \cdot \nabla \Delta_{q} u_{p} u_{q} d x \\
= & I_{111}+I_{112}+I_{113} .
\end{aligned}
$$

Thanks to the facts $\sum_{q-2 \leq p \leq q+2} \Delta_{q} u_{p}=u_{q}$ and $\nabla \cdot u_{\leq q-2}=0$, the term $I_{112}$ vanishes. Notice that $I_{12}$ and $I_{13}$ can be treated in the analogous way as $I_{111}$ and $I_{113}$, respectively. Thus we will only show the estimates of $I_{111}$ and $I_{113}$. Applying the commutator estimate in Lemma 2.3 and Bernstein's inequality to $I_{111}$ gives rise to

$$
\begin{aligned}
\left|I_{111}\right| & \leq \sum_{q \geq-1} \sum_{|p-q| \leq 2} \lambda_{q}^{2 s}\left\|\nabla u_{\leq p-2}\right\|_{\infty}\left\|u_{p}\right\|_{2}\left\|u_{q}\right\|_{2} \\
& \lesssim \sum_{q \geq-1} \lambda_{q}^{2 s}\left\|u_{q}\right\|_{2}^{2} \sum_{p \leq q} \lambda_{p}^{\frac{n}{2}+1}\left\|u_{p}\right\|_{2} \\
& \lesssim \sum_{q \geq-1} \lambda_{q}^{(s+1) \theta}\left\|u_{q}\right\|_{2}^{\theta} \lambda_{q}^{s(2-\theta)}\left\|u_{q}\right\|_{2}^{2-\theta} \sum_{p \leq q} \lambda_{p}^{(s+1) \delta}\left\|u_{p}\right\|_{2}^{\delta} \lambda_{p}^{s(1-\delta)}\left\|u_{p}\right\|_{2}^{1-\delta}\left(\lambda_{q}^{-\theta} \lambda_{p}^{\frac{n}{2}+1-s-\delta}\right) \\
& \lesssim \sum_{q \geq-1} \lambda_{q}^{(s+1) \theta}\left\|u_{q}\right\|_{2}^{\theta} \lambda_{q}^{s(2-\theta)}\left\|u_{q}\right\|_{2}^{2-\theta} \sum_{p \leq q} \lambda_{p}^{(s+1) \delta}\left\|u_{p}\right\|_{2}^{\delta} \lambda_{p}^{s(1-\delta)}\left\|u_{p}\right\|_{2}^{1-\delta} \lambda_{p-q}^{\theta}
\end{aligned}
$$

with constants $\theta$ and $\delta$ satisfying $0<\theta<2,0<\delta<1$ and

$$
s \geq \frac{n}{2}+1-\theta-\delta
$$

It then follows from Young's inequality with $\left(r_{1}, r_{2}, r_{3}, r_{4}\right) \in(1, \infty)^{4}$ satisfying

$$
\frac{1}{r_{1}}+\frac{1}{r_{2}}+\frac{1}{r_{3}}+\frac{1}{r_{4}}=1, \quad r_{1}=\frac{2}{\theta}, \quad r_{3}=\frac{2}{\delta}
$$


such that for some $\theta_{1}>0, \theta_{2}>0$

$$
\begin{aligned}
\left|I_{111}\right| \leq & \frac{\nu}{64} \sum_{q \geq-1} \lambda_{q}^{2 s+2}\left\|u_{q}\right\|_{2}^{2}+C_{\nu} \sum_{q \geq-1}\left(\lambda_{q}^{2 s}\left\|u_{q}\right\|_{2}^{2}\right)^{\frac{(2-\theta) r_{2}}{2}} \\
& +\frac{\nu}{64} \sum_{q \geq-1} \sum_{p \leq q} \lambda_{p}^{2 s+2}\left\|u_{p}\right\|_{2}^{2} \lambda_{p-q}^{\theta_{1}}+C_{\nu} \sum_{q \geq-1} \sum_{p \leq q}\left(\lambda_{p}^{2 s}\left\|u_{p}\right\|_{2}^{2}\right)^{\frac{(1-\delta) r_{4}}{2}} \lambda_{p-q}^{\theta_{2}} \\
\leq & \frac{\nu}{32} \sum_{q \geq-1} \lambda_{q}^{2 s+2}\left\|u_{q}\right\|_{2}^{2}+C_{\nu}\left(\sum_{q \geq-1} \lambda_{q}^{2 s}\left\|u_{q}\right\|_{2}^{2}\right)^{\frac{(2-\theta) r_{2}}{2}}+C_{\nu}\left(\sum_{q \geq-1} \lambda_{q}^{2 s}\left\|u_{q}\right\|_{2}^{2}\right)^{\frac{(1-\delta) r_{4}}{2}}
\end{aligned}
$$

Notice that (3.13) and (3.14) imply that $s>\frac{n}{2}-1$.

To estimate $I_{113}$, it follows from Hölder, Bernstein and Young's inequalities that

$$
\begin{aligned}
\left|I_{113}\right| & \leq \sum_{q \geq-1} \sum_{|p-q| \leq 2} \lambda_{q}^{2 s}\left\|u_{\leq p-2}-u_{\leq q-2}\right\|_{2}\left\|\nabla u_{p}\right\|_{\infty}\left\|u_{q}\right\|_{2} \\
& \lesssim \sum_{q \geq-1} \lambda_{q}^{2 s+\frac{n}{2}+1}\left\|u_{q}\right\|_{2}^{3} \\
& \lesssim \sum_{q \geq-1} \lambda_{q}^{(s+1) \theta}\left\|u_{q}\right\|_{2}^{\theta} \lambda_{q}^{s(3-\theta)}\left\|u_{q}\right\|_{2}^{3-\theta} \lambda_{q}^{\frac{n}{2}+1-s-\theta} \\
& \leq \frac{\nu}{32} \sum_{q \geq-1} \lambda_{q}^{2 s+2}\left\|u_{q}\right\|_{2}^{2}+C_{\nu}\left(\sum_{q \geq-1} \lambda_{q}^{2 s}\left\|u_{q}\right\|_{2}^{2}\right)^{\frac{3-\theta}{2-\theta}}
\end{aligned}
$$

for $s \geq \frac{n}{2}+1-\theta$ and $0<\theta<2$. Thus

$$
I_{1} \leq \frac{\nu}{8}\|\nabla u\|_{H^{s}}^{2}+C_{\nu}\|u\|_{H^{s}}^{2+\gamma_{1}}+C_{\nu}\|u\|_{H^{s}}^{2+\gamma_{2}}
$$

for $s>\frac{n}{2}-1$ and some $\gamma_{1}, \gamma_{2}>0$.

Lemma 3.3. Let $\frac{n}{2}+s-2 r \leq 0$ and $s<r$. The following estimate holds

$$
\left|I_{2}\right| \leq \frac{\nu}{8} \sum_{q \geq-1} \lambda_{q}^{2 s+2}\left\|u_{q}\right\|_{2}^{2}+C_{\nu}\|b\|_{H^{r}}^{4} .
$$

Proof: We first decompose $I_{2}$ by using Bony's paraproduct,

$$
\begin{aligned}
I_{2}= & -\sum_{q \geq-1} \sum_{|q-p| \leq 2} \lambda_{q}^{2 s} \int_{\mathbb{R}^{3}} \Delta_{q}\left(b_{\leq p-2} \cdot \nabla b_{p}\right) u_{q} d x \\
& -\sum_{q \geq-1} \sum_{|q-p| \leq 2} \lambda_{q}^{2 s} \int_{\mathbb{R}^{3}} \Delta_{q}\left(b_{p} \cdot \nabla b_{\leq p-2}\right) u_{q} d x \\
& -\sum_{q \geq-1} \sum_{p \geq q-2} \lambda_{q}^{2 s} \int_{\mathbb{R}^{3}} \Delta_{q}\left(b_{p} \cdot \nabla \tilde{b}_{p}\right) u_{q} d x \\
= & I_{21}+I_{22}+I_{23} .
\end{aligned}
$$


Due to the lack of cancelation, $I_{21}$ is the worst term which can be estimated as

$$
\begin{aligned}
\left|I_{21}\right| & \leq \sum_{q \geq-1} \sum_{|q-p| \leq 2} \lambda_{q}^{2 s+1}\left\|b_{\leq p-2}\right\|_{\infty}\left\|b_{p}\right\|_{2}\left\|_{u_{q}}\right\|_{2} \\
& \lesssim \sum_{q \geq-1} \lambda_{q}^{2 s+1}\left\|b_{q}\right\|_{2}\left\|u_{q}\right\|_{2} \sum_{p \leq q} \lambda_{p}^{\frac{n}{2}}\left\|b_{p}\right\|_{2} \\
& \lesssim \sum_{q \geq-1} \lambda_{q}^{s+1}\left\|u_{q}\right\|_{2} \lambda_{q}^{r}\left\|b_{q}\right\|_{2} \sum_{p \leq q} \lambda_{p}^{r}\left\|b_{p}\right\|_{2} \lambda_{q-p}^{s-r} \lambda_{p}^{\frac{n}{2}+s-2 r} \\
& \lesssim \sum_{q \geq-1} \lambda_{q}^{s+1}\left\|u_{q}\right\|_{2} \lambda_{q}^{r}\left\|b_{q}\right\|_{2} \sum_{p \leq q} \lambda_{p}^{r}\left\|b_{p}\right\|_{2} \lambda_{q-p}^{s-r}
\end{aligned}
$$

for $\frac{n}{2}+s-2 r \leq 0$. As a result, Young's inequality gives rise to

$$
\left|I_{21}\right| \leq \frac{\nu}{16} \sum_{q \geq-1} \lambda_{q}^{2 s+2}\left\|u_{q}\right\|_{2}^{2}+C_{\nu} \sum_{q \geq-1}\left(\lambda_{q}^{r}\left\|b_{q}\right\|_{2} \sum_{p \leq q} \lambda_{p}^{r}\left\|b_{p}\right\|_{2} \lambda_{q-p}^{s-r}\right)^{2} .
$$

Then we apply Jensen's inequality, if $s<r$,

$$
\begin{aligned}
\left|I_{21}\right| & \leq \frac{\nu}{16} \sum_{q \geq-1} \lambda_{q}^{2 s+2}\left\|u_{q}\right\|_{2}^{2}+C_{\nu} \sum_{q \geq-1} \lambda_{q}^{2 r}\left\|b_{q}\right\|_{2}^{2} \sum_{p \leq q} \lambda_{p}^{2 r}\left\|b_{p}\right\|_{2}^{2} \lambda_{q-p}^{s-r} \\
& \leq \frac{\nu}{16} \sum_{q \geq-1} \lambda_{q}^{2 s+2}\left\|u_{q}\right\|_{2}^{2}+C_{\nu}\left(\sum_{q \geq-1} \lambda_{q}^{2 r}\left\|b_{q}\right\|_{2}^{2}\right)^{2} .
\end{aligned}
$$

We claim that $I_{22}$ shares the same estimate as $I_{21}$. Indeed, the following inequality holds

$$
\left|I_{22}\right| \lesssim \sum_{q \geq-1} \lambda_{q}^{2 s}\left\|b_{q}\right\|_{2}\left\|u_{q}\right\|_{2} \sum_{p \leq q} \lambda_{p}^{\frac{n}{2}+1}\left\|b_{p}\right\|_{2} \lesssim\left|I_{21}\right|
$$

To move the derivative from high modes to low modes in $I_{23}$, we apply integration by parts

$$
\left|I_{23}\right|=\left|\sum_{q \geq-1} \sum_{p \geq q-2} \lambda_{q}^{2 s} \int_{\mathbb{R}^{3}} \Delta_{q}\left(b_{p} \otimes \tilde{b}_{p}\right) \cdot \nabla u_{q} d x\right| .
$$

It then follows from Hölder's and Bernstein's inequalities

$$
\begin{aligned}
\left|I_{23}\right| & \lesssim \sum_{q \geq-1} \lambda_{q}^{2 s+1}\left\|u_{q}\right\|_{2} \sum_{p \geq q-4}\left\|b_{p}\right\|_{2}\left\|b_{p}\right\|_{\infty} \\
& \lesssim \sum_{q \geq-1} \lambda_{q}^{2 s+1}\left\|u_{q}\right\|_{2} \sum_{p \geq q-4} \lambda_{p}^{\frac{n}{2}}\left\|b_{p}\right\|_{2}^{2} \\
& \lesssim \sum_{q \geq-1} \lambda_{q}^{s+1}\left\|u_{q}\right\|_{2} \sum_{p \geq q-4} \lambda_{p}^{2 r}\left\|b_{p}\right\|_{2}^{2} \lambda_{q-p}^{s} \lambda_{p}^{\frac{n}{2}+s-2 r} \\
& \lesssim \sum_{q \geq-1} \lambda_{q}^{s+1}\left\|u_{q}\right\|_{2} \sum_{p \geq q-4} \lambda_{p}^{2 r}\left\|b_{p}\right\|_{2}^{2} \lambda_{q-p}^{s}
\end{aligned}
$$


for $\frac{n}{2}+s-2 r \leq 0$. Applying Young's inequality, Jensen's inequality and changing order of the summations yields

$$
\begin{aligned}
\left|I_{23}\right| & \leq \frac{\nu}{16} \sum_{q \geq-1} \lambda_{q}^{2 s+2}\left\|u_{q}\right\|_{2}^{2}+C_{\nu} \sum_{q \geq-1}\left(\sum_{p \geq q-4} \lambda_{p}^{2 r}\left\|b_{p}\right\|_{2}^{2} \lambda_{q-p}^{s}\right)^{2} \\
& \leq \frac{\nu}{16} \sum_{q \geq-1} \lambda_{q}^{2 s+2}\left\|u_{q}\right\|_{2}^{2}+C_{\nu} \sum_{q \geq-1} \sum_{p \geq q-4} \lambda_{p}^{4 r}\left\|b_{p}\right\|_{2}^{4} \lambda_{q-p}^{s} \\
& \leq \frac{\nu}{16} \sum_{q \geq-1} \lambda_{q}^{2 s+2}\left\|u_{q}\right\|_{2}^{2}+C_{\nu} \sum_{p \geq-1} \lambda_{p}^{4 r}\left\|b_{p}\right\|_{2}^{4} \sum_{q \leq p+4} \lambda_{q-p}^{s} \\
& \leq \frac{\nu}{16} \sum_{q \geq-1} \lambda_{q}^{2 s+2}\left\|u_{q}\right\|_{2}^{2}+C_{\nu}\left(\sum_{q \geq-1} \lambda_{q}^{2 r}\left\|b_{q}\right\|_{2}^{2}\right)^{2} .
\end{aligned}
$$

It completes the proof.

Lemma 3.4. Let $s>\frac{n}{2}-1$ and $\frac{n}{4}+\frac{s}{2}<r<s+2-\varepsilon$ with small enough $\varepsilon>0$. We have the estimate

$$
\left|I_{3}\right| \leq \frac{\nu}{8} \sum_{q \geq-1} \lambda_{q}^{2 s+2}\left\|u_{q}\right\|_{2}^{2}+\frac{\mu}{8} \sum_{q \geq-1} \lambda_{q}^{2 r+2}\left\|b_{q}\right\|_{2}^{2}+C_{\nu, \mu}\|u\|_{H^{s}}^{2+\gamma_{3}}+C_{\nu, \mu}\|b\|_{H^{r}}^{2+\gamma_{4}}
$$

for some constants $\gamma_{3}, \gamma_{4}>0$.

Proof: As for $I_{1}$, we first decompose $I_{3}$ by Bony's paraproduct

$$
\begin{aligned}
I_{3}= & \sum_{q \geq-1} \sum_{|q-p| \leq 2} \lambda_{q}^{2 r} \int_{\mathbb{R}^{3}} \Delta_{q}\left(u_{\leq p-2} \cdot \nabla b_{p}\right) b_{q} d x \\
& +\sum_{q \geq-1} \sum_{|q-p| \leq 2} \lambda_{q}^{2 r} \int_{\mathbb{R}^{3}} \Delta_{q}\left(u_{p} \cdot \nabla b_{\leq p-2}\right) b_{q} d x \\
& +\sum_{q \geq-1} \sum_{p \geq q-2} \lambda_{q}^{2 r} \int_{\mathbb{R}^{3}} \Delta_{q}\left(u_{p} \cdot \nabla \tilde{b}_{p}\right) b_{q} d x \\
= & I_{31}+I_{32}+I_{33},
\end{aligned}
$$

and further decompose $I_{31}$ by using the commutator to

$$
\begin{aligned}
I_{31}= & -\sum_{q \geq-1} \sum_{|q-p| \leq 2} \lambda_{q}^{2 r} \int_{\mathbb{R}^{3}}\left[\Delta_{q}, u_{\leq p-2} \cdot \nabla\right] b_{p} b_{q} d x \\
& -\sum_{q \geq-1} \sum_{|q-p| \leq 2} \lambda_{q}^{2 r} \int_{\mathbb{R}^{3}} u_{\leq q-2} \cdot \nabla \Delta_{q} b_{p} b_{q} d x \\
& -\sum_{q \geq-1} \sum_{|q-p| \leq 2} \lambda_{q}^{2 r} \int_{\mathbb{R}^{3}}\left(u_{\leq p-2}-u_{\leq q-2}\right) \cdot \nabla \Delta_{q} b_{p} b_{q} d x \\
= & I_{311}+I_{312}+I_{313} .
\end{aligned}
$$


MIMI DAI

It is not hard to see that $I_{312}=0$. By the commutator estimate in Lemma 2.3, we infer

$$
\begin{aligned}
\left|I_{311}\right| & \leq \sum_{q \geq-1} \sum_{|p-q| \leq 2} \lambda_{q}^{2 r}\left\|\nabla u_{\leq p-2}\right\|_{\infty}\left\|b_{p}\right\|_{2}\left\|b_{q}\right\|_{2} \\
& \lesssim \sum_{q \geq-1} \lambda_{q}^{2 r}\left\|b_{q}\right\|_{2}^{2} \sum_{p \leq q} \lambda_{p}^{\frac{n}{2}+1}\left\|u_{p}\right\|_{2} \\
& \lesssim \sum_{q \geq-1} \lambda_{q}^{(r+1) \theta}\left\|b_{q}\right\|_{2}^{\theta} \lambda_{q}^{r(2-\theta)}\left\|b_{q}\right\|_{2}^{2-\theta} \sum_{p \leq q} \lambda_{p}^{(s+1) \delta}\left\|u_{p}\right\|_{2}^{\delta} \lambda_{p}^{s(1-\delta)}\left\|u_{p}\right\|_{2}^{1-\delta}\left(\lambda_{q}^{-\theta} \lambda_{p}^{\frac{n}{2}+1-s-\delta}\right) \\
& \lesssim \sum_{q \geq-1} \lambda_{q}^{(r+1) \theta}\left\|b_{q}\right\|_{2}^{\theta} \lambda_{q}^{r(2-\theta)}\left\|b_{q}\right\|_{2}^{2-\theta} \sum_{p \leq q} \lambda_{p}^{(s+1) \delta}\left\|u_{p}\right\|_{2}^{\delta} \lambda_{p}^{s(1-\delta)}\left\|u_{p}\right\|_{2}^{1-\delta} \lambda_{p-q}^{\theta}
\end{aligned}
$$

for parameters $\theta$ and $\delta$ satisfying $0<\theta<2,0<\delta<1$ and

$$
s \geq \frac{n}{2}+1-\theta-\delta \text {. }
$$

It then follows from Young's inequality with $\left(r_{1}, r_{2}, r_{3}, r_{4}\right) \in(1, \infty)^{4}$ satisfying

$$
\frac{1}{r_{1}}+\frac{1}{r_{2}}+\frac{1}{r_{3}}+\frac{1}{r_{4}}=1, \quad r_{1}=\frac{2}{\theta}, \quad r_{3}=\frac{2}{\delta}
$$

such that

$$
\begin{aligned}
\left|I_{311}\right| \leq & \frac{\nu}{32} \sum_{q \geq-1} \lambda_{q}^{2 s+2}\left\|u_{q}\right\|_{2}^{2}+\frac{\mu}{32} \sum_{q \geq-1} \lambda_{q}^{2 r+2}\left\|b_{q}\right\|_{2}^{2} \\
& +C_{\nu, \mu}\left(\sum_{q \geq-1} \lambda_{q}^{2 s}\left\|u_{q}\right\|_{2}^{2}\right)^{1+\gamma_{3}}+C_{\nu, \mu}\left(\sum_{q \geq-1} \lambda_{q}^{2 r}\left\|b_{q}\right\|_{2}^{2}\right)^{1+\gamma_{4}}
\end{aligned}
$$

for some constants $\gamma_{3}, \gamma_{4}>0$. Notice that (3.15) and (3.16) imply for large enough $r_{2}$ and $r_{4}$, and $\delta, \theta$ close enough to 1 , there exists a small $\varepsilon>0$ such that

$$
s \geq \frac{n}{2}-\theta+\varepsilon>\frac{n}{2}-1 .
$$

We observe that $\left|I_{313}\right| \lesssim\left|I_{311}\right|$, and hence $I_{313}$ enjoys the same estimate of $I_{311}$.

Following similar strategy as for $I_{311}$, we estimate $I_{32}$ as follows,

$$
\begin{aligned}
\left|I_{32}\right| \leq & \sum_{q \geq-1} \sum_{|q-p| \leq 2} \lambda_{q}^{2 r}\left\|u_{p}\right\|_{2}\left\|\nabla b_{\leq p-2}\right\|_{\infty}\left\|b_{q}\right\|_{2} \\
& \lesssim \sum_{q \geq-1} \lambda_{q}^{2 r}\left\|u_{q}\right\|_{2}\left\|b_{q}\right\|_{2} \sum_{p \leq q} \lambda_{p}^{\frac{n}{2}+1}\left\|b_{p}\right\|_{2} \\
& \lesssim \sum_{q \geq-1} \lambda_{q}^{s+1}\left\|u_{q}\right\|_{2} \lambda_{q}^{(r+1) \theta}\left\|b_{q}\right\|_{2}^{\theta} \lambda_{q}^{r(1-\theta)}\left\|b_{q}\right\|_{2}^{1-\theta} . \\
& \sum_{p \leq q} \lambda_{p}^{r}\left\|b_{p}\right\|_{2} \lambda_{q-p}^{r-s-1-\theta} \lambda_{p}^{\frac{n}{2}-s-\theta} \\
\lesssim & \sum_{q \geq-1} \lambda_{q}^{s+1}\left\|u_{q}\right\|_{2} \lambda_{q}^{(r+1) \theta}\left\|b_{q}\right\|_{2}^{\theta} \lambda_{q}^{r(1-\theta)}\left\|b_{q}\right\|_{2}^{1-\theta} \cdot \sum_{p \leq q} \lambda_{p}^{r}\left\|b_{p}\right\|_{2} \lambda_{q-p}^{r-s-1-\theta}
\end{aligned}
$$

for $0<\theta<1$ and

$$
s \geq \frac{n}{2}-\theta .
$$


It then follows from Young's inequality and Jensen's inequality, with the triplet $\left(2, \frac{2}{\theta}, \frac{2}{1-\theta}\right)$ satisfying

$$
r-s-1-\theta<0
$$

such that

$$
\left|I_{32}\right| \lesssim \frac{\nu}{32} \sum_{q \geq-1} \lambda_{q}^{2 s+2}\left\|u_{q}\right\|_{2}^{2}+\frac{\mu}{32} \sum_{q \geq-1} \lambda_{q}^{2 s+2}\left\|b_{q}\right\|_{2}^{2}+C_{\nu, \mu}\left(\sum_{q \geq-1} \lambda_{p}^{2 r}\left\|b_{p}\right\|_{2}^{2}\right)^{\frac{1}{1-\theta}} .
$$

The constraints (3.17) and (3.18) implies that for $\theta=1-\varepsilon$

$$
s>r-1-\theta, \quad s \geq \frac{n}{2}-1+\varepsilon>\frac{n}{2}-1 .
$$

The term $I_{33}$ can be estimated in an analogous way as for $I_{23}$. To not over burden the analysis with computations, we omit the details and claim

$$
\left|I_{33}\right| \leq \frac{\nu}{32} \sum_{q \geq-1} \lambda_{q}^{2 s+2}\left\|u_{q}\right\|_{2}^{2}+\frac{\mu}{32} \sum_{q \geq-1} \lambda_{q}^{2 s+2}\left\|b_{q}\right\|_{2}^{2}+C_{\nu, \mu}\left(\sum_{q \geq-1} \lambda_{p}^{2 r}\left\|b_{p}\right\|_{2}^{2}\right)^{1+\gamma_{4} / 2}
$$

for some constant $\gamma_{4}>0$.

Lemma 3.5. Let the index $r$ and s satisfy conditions in Lemma 3.4. In addition, assume $r \leq s+1-\varepsilon$ for a small enough constant $\varepsilon>0$. We have

$$
\begin{aligned}
\left|I_{4}\right| \leq & \frac{\nu}{32} \sum_{q \geq-1} \lambda_{q}^{2 s+2}\left\|u_{q}\right\|_{2}^{2}+\frac{\mu}{32} \sum_{q \geq-1} \lambda_{q}^{2 r+2}\left\|b_{q}\right\|_{2}^{2} \\
& +C_{\nu, \mu}\|u\|_{H^{s}}^{2+\gamma_{5}}+C_{\nu, \mu}\|b\|_{H^{r}}^{2+\gamma_{6}}+C_{\nu, \mu}\|b\|_{H^{r}}^{2+\gamma_{7}}
\end{aligned}
$$

for various constants $C_{\nu, \mu}$ depending on $\nu, \mu$, and some constants $\gamma_{5}, \gamma_{6}, \gamma_{7}>0$.

Proof: As usual, using Bony's paraproduct, $I_{4}$ can be written as

$$
\begin{aligned}
I_{4}= & -\sum_{q \geq-1} \sum_{|q-p| \leq 2} \lambda_{q}^{2 r} \int_{\mathbb{R}^{3}} \Delta_{q}\left(b_{\leq p-2} \cdot \nabla u_{p}\right) b_{q} d x \\
& -\sum_{q \geq-1} \sum_{|q-p| \leq 2} \lambda_{q}^{2 r} \int_{\mathbb{R}^{3}} \Delta_{q}\left(b_{p} \cdot \nabla u_{\leq p-2}\right) b_{q} d x \\
& -\sum_{q \geq-1} \sum_{p \geq q-2} \lambda_{q}^{2 r} \int_{\mathbb{R}^{3}} \Delta_{q}\left(\tilde{b}_{p} \cdot \nabla u_{p}\right) b_{q} d x \\
= & I_{41}+I_{42}+I_{43} .
\end{aligned}
$$

First we notice that $I_{42}$ and $I_{43}$ can be estimated as $I_{311}$ and $I_{33}$, respectively. While $I_{41}$ needs to be treated in a different way, since cancellation is not available 
here. Applying Hölder's inequality and Bernstein's inequality first, we get

$$
\begin{aligned}
\left|I_{41}\right| \leq & \sum_{q \geq-1} \sum_{|q-p| \leq 2} \lambda_{q}^{2 r}\left\|b_{\leq p-2}\right\|_{\infty}\left\|\nabla u_{p}\right\|_{2}\left\|b_{q}\right\|_{2} \\
\lesssim & \sum_{q \geq-1} \lambda_{q}^{2 r+1}\left\|b_{q}\right\|_{2}\left\|u_{q}\right\|_{2} \sum_{p \leq q}\left\|b_{p}\right\|_{\infty} \\
\lesssim & \sum_{q \geq-1} \lambda_{q}^{2 r+1}\left\|b_{q}\right\|_{2}\left\|u_{q}\right\|_{2} \sum_{p \leq q} \lambda_{p}^{\frac{n}{2}}\left\|b_{p}\right\|_{2} \\
\lesssim & \sum_{q \geq-1}\left(\lambda_{q}^{(r+1) \delta}\left\|b_{q}\right\|_{2}^{\delta}\right)\left(\lambda_{q}^{r(1-\delta)}\left\|b_{q}\right\|_{2}^{1-\delta}\right)\left(\lambda_{q}^{(s+1) \eta}\left\|u_{q}\right\|_{2}^{\eta}\right)\left(\lambda_{q}^{s(1-\eta)}\left\|u_{q}\right\|_{2}^{1-\eta}\right) \\
& \cdot\left(\sum_{p \leq q} \lambda_{p}^{r}\left\|b_{p}\right\|_{2} \lambda_{q-p}^{r+1-s-\delta-\eta} \lambda_{p}^{\frac{n}{2}+1-s-\delta-\eta}\right) \\
\lesssim & \sum_{q \geq-1}\left(\lambda_{q}^{(r+1) \delta}\left\|b_{q}\right\|_{2}^{\delta}\right)\left(\lambda_{q}^{r(1-\delta)}\left\|b_{q}\right\|_{2}^{1-\delta}\right)\left(\lambda_{q}^{(s+1) \eta}\left\|u_{q}\right\|_{2}^{\eta}\right)\left(\lambda_{q}^{s(1-\eta)}\left\|u_{q}\right\|_{2}^{1-\eta}\right) \\
& \cdot\left(\sum_{p \leq q} \lambda_{p}^{r}\left\|b_{p}\right\|_{2} \lambda_{q-p}^{r+1-s-\delta-\eta}\right)
\end{aligned}
$$

provided that $\frac{n}{2}+1-s-\delta-\eta \leq 0$. We apply Young's inequality with parameters $1 \leq r_{1}, r_{2}, r_{3}, r_{4}, r_{5} \leq \infty$ satisfying

$$
\frac{1}{r_{1}}+\frac{1}{r_{2}}+\frac{1}{r_{3}}+\frac{1}{r_{4}}+\frac{1}{r_{5}}=1, \quad r_{1}=\frac{2}{\delta}, \quad r_{3}=\frac{2}{\eta},
$$

for some $\delta, \eta \in(0,1)$. It yields that

$$
\begin{aligned}
\left|I_{41}\right| \leq & \frac{\nu}{64} \sum_{q \geq-1} \lambda_{q}^{2 s+2}\left\|u_{q}\right\|_{2}^{2}+\frac{\mu}{64} \sum_{q \geq-1} \lambda_{q}^{2 r+2}\left\|b_{q}\right\|_{2}^{2}+C_{\nu, \mu} \sum_{q \geq-1} \lambda_{q}^{r(1-\delta) r_{2}}\left\|b_{q}\right\|_{2}^{(1-\delta) r_{2}} \\
& +C_{\nu, \mu} \sum_{q \geq-1} \lambda_{q}^{s(1-\eta) r_{4}}\left\|u_{q}\right\|_{2}^{(1-\eta) r_{4}}+C_{\nu, \mu} \sum_{q \geq-1}\left(\sum_{p \leq q} \lambda_{p}^{r}\left\|b_{p}\right\|_{2} \lambda_{q-p}^{r+1-s-\delta-\eta}\right)^{r_{5}} .
\end{aligned}
$$

Assume $r<s-1+\delta+\eta$. Using Jensen's inequality to the last term and exchanging the order of summation gives rise to

$$
\begin{aligned}
\sum_{q \geq-1}\left(\sum_{p \leq q} \lambda_{p}^{r}\left\|b_{p}\right\|_{2} \lambda_{q-p}^{r+1-s-\delta-\eta}\right)^{r_{5}} & \lesssim \sum_{q \geq-1} \sum_{p \leq q} \lambda_{p}^{r r_{5}}\left\|b_{p}\right\|_{2}^{r_{5}} \lambda_{q-p}^{r+1-s-\delta-\eta} \\
& \lesssim \sum_{p \leq-1} \lambda_{p}^{r r_{5}}\left\|b_{p}\right\|_{2}^{r_{5}} \sum_{q \geq p} \lambda_{q-p}^{r+1-s-\delta-\eta} \\
& \lesssim\left(\sum_{p \leq-1} \lambda_{p}^{2 r}\left\|b_{p}\right\|_{2}^{2}\right)^{\frac{r_{5}}{2}} .
\end{aligned}
$$

Thus one can choose $\delta$ and $\eta$ close enough to 1 and $r_{2}, r_{4}, r_{5}$ large enough such that $(1-\delta) r_{2}=2+\gamma_{5},(1-\eta) r_{4}=2+\gamma_{6}$ and $r_{5} / 2=1+\gamma_{7} / 2$ with $\gamma_{5}, \gamma_{6}, \gamma_{7}>0$. It 
then follows that

$$
\begin{aligned}
\left|I_{41}\right| \leq & \frac{\nu}{64} \sum_{q \geq-1} \lambda_{q}^{2 s+2}\left\|u_{q}\right\|_{2}^{2}+\frac{\mu}{64} \sum_{q \geq-1} \lambda_{q}^{2 r+2}\left\|b_{q}\right\|_{2}^{2} \\
& +C_{\nu, \mu}\|u\|_{H^{s}}^{2+\gamma_{5}}+C_{\nu, \mu}\|b\|_{H^{r}}^{2+\gamma_{6}}+C_{\nu, \mu}\|b\|_{H^{r}}^{2+\gamma_{7}}
\end{aligned}
$$

Indeed, one can choose $\delta+\eta=2-\varepsilon$ with $\varepsilon=\frac{1}{2}\left[s-\left(\frac{n}{2}-1\right)\right]$.

Lemma 3.6. Let $r>\frac{n}{2}$. Then $I_{5}$ satisfies

$$
\left|I_{5}\right| \leq \frac{\mu}{16} \sum_{q \geq-1} \lambda_{q}^{2 r+2}\left\|b_{q}\right\|_{2}^{2}+C_{\mu}\|b\|_{H^{r}}^{2+\gamma_{8}}+C_{\mu}\|b\|_{H^{r}}^{2+\gamma_{9}}
$$

for some constants $\gamma_{8}, \gamma_{9}>0$.

Proof: Applying Bony's paraproduct first, we decompose $I_{5}$ to

$$
\begin{aligned}
I_{5}= & \sum_{q \geq-1} \sum_{|q-p| \leq 2} \lambda_{q}^{2 r} \int_{\mathbb{R}^{3}} \Delta_{q}\left(b_{\leq p-2} \times\left(\nabla \times b_{p}\right)\right) \cdot \nabla \times b_{q} d x \\
& +\sum_{q \geq-1} \sum_{|q-p| \leq 2} \lambda_{q}^{2 r} \int_{\mathbb{R}^{3}} \Delta_{q}\left(b_{p} \times\left(\nabla \times b_{\leq p-2}\right)\right) \cdot \nabla \times b_{q} d x \\
& +\sum_{q \geq-1} \sum_{p \geq q-2} \lambda_{q}^{2 r} \int_{\mathbb{R}^{3}} \Delta_{q}\left(b_{p} \times\left(\nabla \times \tilde{b}_{p}\right)\right) \cdot \nabla \times b_{q} d x \\
= & I_{51}+I_{52}+I_{53} .
\end{aligned}
$$

Using the commutator notation (2.9), $I_{51}$ can be further decomposed as

$$
\begin{aligned}
I_{51}= & \sum_{q \geq-1} \sum_{|q-p| \leq 2} \lambda_{q}^{2 r} \int_{\mathbb{R}^{3}}\left[\Delta_{q}, b_{\leq p-2} \times \nabla \times\right] b_{p} \cdot \nabla \times b_{q} d x \\
& +\sum_{q \geq-1} \lambda_{q}^{2 r} \int_{\mathbb{R}^{3}} b_{\leq q-2} \times\left(\nabla \times b_{q}\right) \cdot \nabla \times b_{q} d x \\
& +\sum_{q \geq-1} \sum_{|p-q| \leq 2} \lambda_{q}^{2 r} \int_{\mathbb{R}^{3}}\left(b_{\leq p-2}-b_{\leq q-2}\right) \times\left(\nabla \times\left(b_{p}\right)_{q}\right) \cdot \nabla \times b_{q} d x \\
= & I_{511}+I_{512}+I_{513},
\end{aligned}
$$

where we used the fact $\sum_{q-2 \leq p \leq q+2} \Delta_{q} b_{p}=b_{q}$. It is clear that $I_{512}=0$ due to the cross product property. By the commutator estimate in Lemma 2.5, we infer

$$
\begin{aligned}
\left|I_{511}\right| & \lesssim \sum_{q \geq-1} \sum_{|p-q| \leq 2} \lambda_{q}^{2 r+1}\left\|\nabla b_{\leq p-2}\right\|_{\infty}\left\|b_{p}\right\|_{2}\left\|b_{q}\right\|_{2} \\
& \lesssim \sum_{q \geq-1} \lambda_{q}^{2 r+1}\left\|b_{q}\right\|_{2}^{2} \sum_{p \leq q} \lambda_{p}\left\|b_{p}\right\|_{\infty} \\
& \lesssim \sum_{q \geq-1} \lambda_{q}^{2 r+1}\left\|b_{q}\right\|_{2}^{2} \sum_{p \leq q} \lambda_{p}^{1+\frac{n}{2}}\left\|b_{p}\right\|_{2} \\
& \lesssim \sum_{q \geq-1} \lambda_{q}^{(r+1) \theta}\left\|b_{q}\right\|_{2}^{\theta} \lambda_{q}^{r(2-\theta)}\left\|b_{q}\right\|_{2}^{2-\theta} \sum_{p \leq q} \lambda_{p}^{(r+1) \delta}\left\|b_{p}\right\|_{2}^{\delta} \lambda_{p}^{r(1-\delta)}\left\|b_{p}\right\|_{2}^{1-\delta} \lambda_{p}^{1+\frac{n}{2}-r-\delta} \lambda_{q}^{1-\theta} \\
& \lesssim \sum_{q \geq-1} \lambda_{q}^{(r+1) \theta}\left\|b_{q}\right\|_{2}^{\theta} \lambda_{q}^{r(2-\theta)}\left\|b_{q}\right\|_{2}^{2-\theta} \sum_{p \leq q} \lambda_{p}^{(r+1) \delta}\left\|b_{p}\right\|_{2}^{\delta} \lambda_{p}^{r(1-\delta)}\left\|b_{p}\right\|_{2}^{1-\delta} \lambda_{q-p}^{1-\theta}
\end{aligned}
$$


for $0<\theta<2,0<\delta<1$ and

$$
r \geq \frac{n}{2}+2-(\theta+\delta), \quad 1-\theta<0 .
$$

It then follows from Young's inequality with $\left(r_{1}, r_{2}, r_{3}, r_{4}\right) \in(1, \infty)^{4}$ satisfying

$$
\frac{1}{r_{1}}+\frac{1}{r_{2}}+\frac{1}{r_{3}}+\frac{1}{r_{4}}=1, \quad r_{1}=\frac{2}{\theta}, \quad r_{3}=\frac{2}{\delta}
$$

such that

$$
\left|I_{511}\right| \leq \frac{\mu}{16} \sum_{q \geq-1} \lambda_{q}^{2 r+2}\left\|b_{q}\right\|_{2}^{2}+C_{\mu}\left(\sum_{q \geq-1} \lambda_{p}^{2 r}\left\|b_{p}\right\|_{2}^{2}\right)^{1+\bar{\gamma}_{1}}+C_{\mu}\left(\sum_{q \geq-1} \lambda_{p}^{2 r}\left\|b_{p}\right\|_{2}^{2}\right)^{1+\bar{\gamma}_{2}}
$$

for some constants $\bar{\gamma}_{1}, \bar{\gamma}_{2}>0$. The conditions (3.19) and (3.20) imply that

$$
r \geq \frac{n}{2}+2-2+\varepsilon>\frac{n}{2}, \quad \alpha>\frac{1}{\theta}=\frac{1}{2-\varepsilon}>\frac{1}{2}
$$

provided $\theta$ close enough to 2 and $\delta$ close enough to 0 .

The term $I_{513}$ is estimated as follows,

$$
\begin{aligned}
\left|I_{513}\right| & \leq \sum_{q \geq-1} \sum_{|p-q| \leq 2} \lambda_{q}^{2 r} \int_{\mathbb{R}^{3}}\left|\left(b_{\leq p-2}-b_{\leq q-2}\right) \times\left(\nabla \times\left(b_{p}\right)_{q}\right) \cdot \nabla \times b_{q}\right| d x \\
& \lesssim \sum_{q \geq-1} \sum_{|p-q| \leq 2} \lambda_{q}^{2 r}\left\|\nabla b_{q}\right\|_{\infty}\left\|b_{\leq p-2}-b_{\leq q-2}\right\|_{2}\left\|\nabla b_{p}\right\|_{2} \\
& \lesssim \sum_{q \geq-1} \lambda_{q}^{2 r+\frac{n}{2}+2}\left\|b_{q}\right\|_{2}^{3} \\
& \lesssim \sum_{q \geq-1} \lambda_{q}^{(r+1) \theta}\left\|b_{q}\right\|_{2}^{\theta} \lambda_{q}^{r(3-\theta)}\left\|b_{q}\right\|_{2}^{3-\theta} \lambda_{q}^{\frac{n}{2}+2-r-\theta} \\
& \lesssim \sum_{q \geq-1} \lambda_{q}^{(r+1) \theta}\left\|b_{q}\right\|_{2}^{\theta} \lambda_{q}^{r(3-\theta)}\left\|b_{q}\right\|_{2}^{3-\theta}
\end{aligned}
$$

for $0<\theta<2$ and

$$
r \geq \frac{n}{2}+2-\theta=\frac{n}{2}+2-2+\varepsilon>\frac{n}{2}
$$

provided $\theta=2-\varepsilon$ with small enough $\varepsilon$. Thus, we have by Young's inequality that

$$
\left|I_{513}\right| \leq \frac{\mu}{16} \sum_{q \geq-1} \lambda_{q}^{2 r+2}\left\|b_{q}\right\|_{2}^{2}+C_{\mu}\left(\sum_{q \geq-1} \lambda_{p}^{2 r}\left\|b_{p}\right\|_{2}^{2}\right)^{1+\bar{\gamma}_{3}}
$$

for some constant $\bar{\gamma}_{3}>0$.

Notice that

$$
\begin{aligned}
\left|I_{52}\right| & =\left|\sum_{q \geq-1} \sum_{|q-p| \leq 2} \lambda_{q}^{2 r} \int_{\mathbb{R}^{3}} \Delta_{q}\left(\nabla \times b_{\leq p-2} \times b_{p}\right) \cdot \nabla \times b_{q} d x\right| \\
& \lesssim \sum_{q \geq-1} \sum_{|q-p| \leq 2} \lambda_{q}^{2 r+1}\left\|b_{p}\right\|_{2}\left\|\nabla b_{\leq p-2}\right\|_{\infty}\left\|b_{q}\right\|_{2},
\end{aligned}
$$

thus $I_{52}$ enjoys the same estimate as for $I_{511}$. 
To estimate $I_{53}$, we proceed as, by using Hölder's inequality and Bernstein's inequality

$$
\begin{aligned}
\left|I_{53}\right| & \leq \sum_{q \geq-1} \sum_{p \geq q-2} \lambda_{q}^{2 r} \int_{\mathbb{R}^{3}}\left|\Delta_{q}\left(b_{p} \times \nabla \times \tilde{b}_{p}\right) \cdot \nabla \times b_{q}\right| d x \\
& \lesssim \sum_{q \geq-1} \lambda_{q}^{2 r}\left\|\nabla b_{q}\right\|_{\infty} \sum_{p \geq q-3}\left\|b_{p}\right\|_{2}\left\|\nabla b_{p}\right\|_{2} \\
& \lesssim \sum_{q \geq-1} \lambda_{q}^{2 r+1+\frac{n}{2}}\left\|b_{q}\right\|_{2} \sum_{p \geq q-3} \lambda_{p}\left\|b_{p}\right\|_{2}^{2} \\
& \lesssim \sum_{q \geq-1} \lambda_{q}^{(r+1) \theta}\left\|b_{q}\right\|_{2}^{\theta} \lambda_{q}^{r(1-\theta)}\left\|b_{q}\right\|_{2}^{1-\theta} \sum_{p \geq q-3} \lambda_{p}^{(r+1) \delta}\left\|b_{p}\right\|_{2}^{\delta} \lambda_{p}^{r(2-\delta)}\left\|b_{p}\right\|_{2}^{2-\delta} \\
& \cdot \lambda_{p-q}^{1-2 r-\delta} \lambda_{q}^{\frac{n}{2}+2-r-(\theta+\delta)} \\
& \lesssim \sum_{q \geq-1} \lambda_{q}^{(r+1) \theta}\left\|b_{q}\right\|_{2}^{\theta} \lambda_{q}^{r(1-\theta)}\left\|b_{q}\right\|_{2}^{1-\theta} \sum_{p \geq q-3} \lambda_{p}^{(r+1) \delta}\left\|b_{p}\right\|_{2}^{\delta} \lambda_{p}^{r(2-\delta)}\left\|b_{p}\right\|_{2}^{2-\delta} \lambda_{p-q}^{1-2 r-\delta}
\end{aligned}
$$

for $0<\theta<1,0<\delta<2$ and

$$
r \geq \frac{n}{2}+2-(\theta+\delta), \quad 1-2 r-\delta<0 .
$$

Then by Young's inequality with $\left(r_{1}, r_{2}, r_{3}, r_{4}\right) \in(1, \infty)^{4}$ satisfying

$$
\frac{1}{r_{1}}+\frac{1}{r_{2}}+\frac{1}{r_{3}}+\frac{1}{r_{4}}=1, \quad r_{1}=\frac{2}{\theta}, \quad r_{3}=\frac{2}{\delta}
$$

and Jensen's inequality, we have

$$
\left|I_{53}\right| \leq \frac{\mu}{16} \sum_{q \geq-1} \lambda_{q}^{2 r+2}\left\|b_{q}\right\|_{2}^{2}+C_{\mu}\left(\sum_{q \geq-1} \lambda_{p}^{2 r}\left\|b_{p}\right\|_{2}^{2}\right)^{1+\bar{\gamma}_{4}}+C_{\mu}\left(\sum_{q \geq-1} \lambda_{p}^{2 r}\left\|b_{p}\right\|_{2}^{2}\right)^{1+\bar{\gamma}_{5}}
$$

for some constants $\bar{\gamma}_{4}, \bar{\gamma}_{5}>0$. Again, (3.23) and (3.24) imply

$$
r>\frac{n}{2}
$$

provided $r_{2}, r_{4}$ are large enough. To summarize, we have for $r>\frac{n}{2}$

$\left|I_{5}\right| \leq \frac{\mu}{16} \sum_{q \geq-1} \lambda_{q}^{2 r+2}\left\|b_{q}\right\|_{2}^{2}+C_{\mu}\left(\sum_{q \geq-1} \lambda_{p}^{2 r}\left\|b_{p}\right\|_{2}^{2}\right)^{1+\gamma_{8} / 2}+C_{\mu}\left(\sum_{q \geq-1} \lambda_{p}^{2 r}\left\|b_{p}\right\|_{2}^{2}\right)^{1+\gamma_{9} / 2}$

for some constants $\gamma_{8}, \gamma_{9}>0$. In fact, we can take $\gamma_{8} / 2$ as the smallest number of $\bar{\gamma}_{1}, \ldots, \bar{\gamma}_{5}$ and $\gamma_{9} / 2$ as the largest one of these constants.

We are ready to show the uniform estimate for $\|u(t)\|_{H^{s}}^{2}+\|b(t)\|_{H^{r}}^{2}$ on a short time interval.

Lemma 3.7. Assume $r$ and s satisfy

$$
s>\frac{n}{2}-1, \quad r>\frac{n}{2}, \quad \frac{n}{4}+\frac{s}{2}<r \leq s+1-\varepsilon
$$

for a small enough constant $\varepsilon>0$. There exists a time $T=T\left(\nu, \mu,\left\|u_{0}\right\|_{H^{s}},\left\|b_{0}\right\|_{H^{r}}\right)$ and a constant $C_{\nu, \mu}$ depending on $\nu$ and $\mu$ such that

$$
\|u(t)\|_{H^{s}}^{2}+\|b(t)\|_{H^{r}}^{2} \leq C_{\nu, \mu}\left(\left\|u_{0}\right\|_{H^{s}}^{2}+\left\|b_{0}\right\|_{H^{r}}^{2}\right), \quad \forall t \in[0, T] .
$$


Proof: Combining (3.11), (3.12), and the estimates in Lemma 3.2 to Lemma 3.6. there exist various constants $C_{\nu, \mu}$ depending on $\nu$ and $\mu$ such that

$$
\begin{aligned}
& \frac{d}{d t}\left(\|u\|_{H^{s}}^{2}+\|b\|_{H^{r}}^{2}\right)+\nu\|\nabla u\|_{H^{s}}^{2}+\mu\|\nabla b\|_{H^{r}}^{2} \\
\leq & C_{\nu, \mu}\left(\|u\|_{H^{s}}^{2}+\|b\|_{H^{r}}^{2}\right)^{1+\underline{\gamma}}+C_{\nu, \mu}\left(\|u\|_{H^{s}}^{2}+\|b\|_{H^{r}}^{2}\right)^{1+\bar{\gamma}}
\end{aligned}
$$

with constants $\underline{\gamma}=\min \left\{\gamma_{1}, \ldots, \gamma_{9}\right\}$ and $\bar{\gamma}=\max \left\{\gamma_{1}, \ldots, \gamma_{9}\right\}$. Denote $\psi(t)=$ $\|u(t)\|_{H^{s}}^{2}+\| b(t) \overline{\|}_{H^{r}}^{2}$. Let

$$
T=\frac{1}{2} \min \left\{\frac{1}{C_{\nu, \mu} \underline{\gamma} \psi \underline{\underline{\gamma}}(0)}, \frac{1}{C_{\nu, \mu} \bar{\gamma} \psi \bar{\gamma}(0)}\right\} .
$$

It follows from the energy inequality above that for $t \in[0, T]$,

$$
\begin{aligned}
\|u(t)\|_{H^{s}}^{2}+\|b(t)\|_{H^{r}}^{2} \leq & \frac{\left\|u_{0}\right\|_{H^{s}}^{2}+\left\|b_{0}\right\|_{H^{r}}^{2}}{\left[1-\underline{\gamma} C_{\nu, \mu}\left(\left\|u_{0}\right\|_{H^{s}}^{2}+\left\|b_{0}\right\|_{H^{r}}^{2}\right)^{\underline{\gamma}} t\right]^{1 / \underline{\gamma}}} \\
& +\frac{\left\|u_{0}\right\|_{H^{s}}^{2}+\left\|b_{0}\right\|_{H^{r}}^{2}}{\left[1-\bar{\gamma} C_{\nu, \mu}\left(\left\|u_{0}\right\|_{H^{s}}^{2}+\left\|b_{0}\right\|_{H^{r}}^{2}\right)^{\bar{\gamma}} t\right]^{1 / \bar{\gamma}}} .
\end{aligned}
$$

It completes the proof of the lemma and concludes the proof of Theorem 3.1

\section{UNIQUENESS AND CONTINUITY}

In this section, we establish the uniqueness of solutions stated in Theorem 1.1 The continuity in time can be obtained through a rather standard procedure, see 15; hence we omit the proof.

Theorem 4.1. Let $\varepsilon>0$ be small enough. Assume $\left(u_{1}, b_{1}, p_{1}\right)$ and $\left(u_{2}, b_{2}, p_{2}\right)$ are solutions of (1.1)-(1.2) in $H^{s}\left(\mathbb{R}^{n}\right) \times H^{s+1-\varepsilon}\left(\mathbb{R}^{n}\right)$ satisfying the estimates in Theorem 3.1. Then $\left(u_{1}, b_{1}\right)=\left(u_{2}, b_{2}\right)$.

Proof: The difference $(U, B, \pi)=\left(u_{1}-u_{2}, b_{1}-b_{2}, p_{1}-p_{2}\right)$ satisfies the equations

$$
\begin{array}{r}
U_{t}+u_{2} \cdot \nabla U-b_{2} \cdot \nabla B+U \cdot \nabla u_{1}-B \cdot \nabla b_{1}+\nabla \pi=\nu \Delta U, \\
B_{t}+u_{2} \cdot \nabla B-b_{2} \cdot \nabla U+U \cdot \nabla b_{1}-B \cdot \nabla u_{1}-\nabla \times\left(\left(\nabla \times b_{2}\right) \times B\right) \\
+\nabla \times\left((\nabla \times B) \times b_{1}\right)=\mu \Delta B .
\end{array}
$$

The goal is to obtain a Grönwall type of inequality for the $L^{2}$ energy of $(U, B)$. Thus, we take inner product of the equations of $U$ and $B$ in (4.25) with $U$ and $B$, respectively, to arrive at

$$
\begin{aligned}
& \frac{d}{d t}\left(\frac{1}{2}\|U\|_{2}^{2}+\frac{1}{2}\|B\|_{2}^{2}\right)+\nu\|\nabla U\|_{2}^{2}+\mu\|\nabla B\|_{2}^{2} \\
= & \int_{\mathbb{R}^{n}}\left(b_{2} \cdot \nabla\right) B \cdot U d x+\int_{\mathbb{R}^{n}}(B \cdot \nabla) b_{1} \cdot U d x-\int_{\mathbb{R}^{n}}\left(u_{2} \cdot \nabla\right) U \cdot U d x \\
& -\int_{\mathbb{R}^{n}}(U \cdot \nabla) u_{1} \cdot U d x+\int_{\mathbb{R}^{n}}\left(b_{2} \cdot \nabla\right) U \cdot B d x+\int_{\mathbb{R}^{n}}(B \cdot \nabla) u_{1} \cdot B d x \\
& -\int_{\mathbb{R}^{n}}\left(u_{2} \cdot \nabla\right) B \cdot B d x-\int_{\mathbb{R}^{n}}(U \cdot \nabla) b_{1} \cdot B d x \\
& +\int_{\mathbb{R}^{n}} \nabla \times\left(\left(\nabla \times b_{2}\right) \times B\right) \cdot B d x-\int_{\mathbb{R}^{n}} \nabla \times\left((\nabla \times B) \times b_{1}\right) \cdot B d x .
\end{aligned}
$$


Since $\left(u_{1}, b_{1}\right)$ and $\left(u_{2}, b_{2}\right)$ are in $H^{s}\left(\mathbb{R}^{n}\right) \times H^{s+1-\varepsilon}\left(\mathbb{R}^{n}\right)$ with $s>\frac{n}{2}-1$, so is $(U, B)$. Thus it can be justified that many terms on the right hand side vanish, i.e.

$$
\begin{array}{r}
\int_{\mathbb{R}^{n}}\left(u_{2} \cdot \nabla\right) U \cdot U d x=0, \quad \int_{\mathbb{R}^{n}}\left(u_{2} \cdot \nabla\right) B \cdot B d x=0, \\
\int_{\mathbb{R}^{n}} \nabla \times\left((\nabla \times B) \times b_{1}\right) \cdot B d x=0 \\
\int_{\mathbb{R}^{n}}\left(b_{2} \cdot \nabla\right) B \cdot U d x+\int_{\mathbb{R}^{n}}\left(b_{2} \cdot \nabla\right) U \cdot B d x=0 .
\end{array}
$$

We are left to estimate the five non-zero flux terms. The first one is estimated as

$$
\begin{aligned}
\left|\int_{\mathbb{R}^{n}}(B \cdot \nabla) b_{1} \cdot U d x\right| & =\left|\int_{\mathbb{R}^{n}}(B \cdot \nabla) U \cdot b_{1} d x\right| \\
& \leq\|B\|_{2}\|\nabla U\|_{2}\left\|b_{1}\right\|_{\infty} \\
& \leq \frac{\nu}{8}\|\nabla U\|_{2}^{2}+C_{\nu}\|B\|_{2}^{2}\left\|b_{1}\right\|_{\infty}^{2} \\
& \leq \frac{\nu}{8}\|\nabla U\|_{2}^{2}+C_{\nu}\|B\|_{2}^{2}\left\|b_{1}\right\|_{H^{s+1-\epsilon}}^{2}
\end{aligned}
$$

where we used the embedding $H^{s+1-\varepsilon} \subset L^{\infty}$ for $s+1-\varepsilon>\frac{n}{2}$ (since we can choose $\varepsilon=\frac{1}{2}\left[s-\left(\frac{n}{2}-1\right)\right]$ and $\left.s>\frac{n}{2}-1\right)$. Analogous computation shows

$$
\begin{aligned}
& \left|\int_{\mathbb{R}^{n}}(U \cdot \nabla) u_{1} \cdot U d x\right| \leq \frac{\nu}{8}\|\nabla U\|_{2}^{2}+C_{\nu}\|U\|_{2}^{2}\left\|u_{1}\right\|_{H^{s+1}}^{2}, \\
& \left|\int_{\mathbb{R}^{n}}(B \cdot \nabla) u_{1} \cdot B d x\right| \leq \frac{\mu}{8}\|\nabla B\|_{2}^{2}+C_{\mu}\|B\|_{2}^{2}\left\|u_{1}\right\|_{H^{s+1}}^{2}, \\
& \left|\int_{\mathbb{R}^{n}}(U \cdot \nabla) b_{1} \cdot B d x\right| \leq \frac{\mu}{8}\|\nabla B\|_{2}^{2}+C_{\mu}\|U\|_{2}^{2}\left\|b_{1}\right\|_{H^{s+1-\varepsilon}}^{2} .
\end{aligned}
$$

In the end, we estimate the Hall term as follows

$$
\begin{aligned}
\left|\int_{\mathbb{R}^{n}} \nabla \times\left(\left(\nabla \times b_{2}\right) \times B\right) \cdot B d x\right| & =\left|\int_{\mathbb{R}^{n}}\left(\left(\nabla \times b_{2}\right) \times B\right) \cdot \nabla \times B d x\right| \\
& \leq\|\nabla \times B\|_{2}\left\|\nabla \times b_{2}\right\|_{\infty}\|B\|_{2} \\
& \leq \frac{\mu}{8}\|\nabla B\|_{2}^{2}+C_{\mu}\left\|\nabla \times b_{2}\right\|_{\infty}^{2}\|B\|_{2}^{2} \\
& \leq \frac{\mu}{8}\|\nabla B\|_{2}^{2}+C_{\mu}\left\|\nabla b_{2}\right\|_{H^{s+1-\varepsilon}}^{2}\|B\|_{2}^{2} .
\end{aligned}
$$

The estimates above along with (4.26) give us

$$
\begin{aligned}
& \frac{d}{d t}\left(\|U\|_{2}^{2}+\|B\|_{2}^{2}\right)+\nu\|\nabla U\|_{2}^{2}+\mu\|\nabla B\|_{2}^{2} \\
\leq & C_{\nu, \mu}\left(\left\|u_{1}\right\|_{H^{s+1}}^{2}+\left\|\nabla b_{2}\right\|_{H^{s+1-\varepsilon}}^{2}+\left\|b_{1}\right\|_{H^{s+1-\varepsilon}}^{2}\right)\left(\|U\|_{2}^{2}+\|B\|_{2}^{2}\right) \\
\leq & C_{\nu, \mu}\left(\left\|u_{1}\right\|_{H^{s+1}}^{2}+\left\|\nabla b_{2}\right\|_{H^{s+1-\varepsilon}}^{2}+C\right)\left(\|U\|_{2}^{2}+\|B\|_{2}^{2}\right) .
\end{aligned}
$$

It follows from Grönwall's inequality that

$$
\begin{aligned}
& \|U(t)\|_{2}^{2}+\|B(t)\|_{2}^{2} \\
\leq & \left(\|U(0)\|_{2}^{2}+\|B(0)\|_{2}^{2}\right) e^{C C_{\nu, \mu} t} \exp \left\{C_{\nu, \mu} \int_{0}^{t}\left\|u_{1}(\tau)\right\|_{H^{s+1}}^{2}+\left\|\nabla b_{2}(\tau)\right\|_{H^{s+1-\varepsilon}}^{2} d \tau\right\} .
\end{aligned}
$$


Since $U(0)=B(0)=0, u_{1} \in L^{2}\left(0, T ; H^{s+1}\right)$ and $b_{2} \in L^{2}\left(0, T ; H^{s+2-\varepsilon}\right)$, we infer

$$
\|U(t)\|_{2}^{2}+\|B(t)\|_{2}^{2}=0, \quad \forall t \in[0, T]
$$

\section{REFERENCES}

[1] M. Acheritogaray, P. Degond, A. Frouvelle and J-G. Liu. Kinetic formulation and global existence for the Hall-Magnetohydrodynamic system. Kinetic and Related Models, 4: 901918, 2011.

[2] H. Bahouri, J. Chemin, and R. Danchin. Fourier analysis and nonlinear partial differential equations. Grundlehrender Mathematischen Wissenschaften, 343. Springer, Heidelberg, 2011.

[3] D. Chae, P. Degond and J-G. Liu. Well-posedness for Hall-magnetohydrodynamics. arXiv:1212.3919, 2012.

[4] D. Chae and J. Lee. On the blow-up criterion and small data global existence for the Hallmagneto-hydrodynamics. J. Differential Equations, 256: 3835-3858, 2014.

[5] D. Chae, and M. Schonbek. On the temporal decay for the Hall-magnetohydrodynamic equations. Journal of Differential Equations, Vol.255, Iss. 11: 3971-3982, 2013.

[6] D. Chae, R. Wan and J. Wu. Local well-posedness for the Hall-MHD equations with fractional magnetic diffusion. Journal of Mathematical Fluid Mechanics, Vol. 17, Iss. 4: 627-638, 2015.

[7] D. Chae and J. Wolf. On partial regularity for the 3D non-stationary Hall magnetohydrodynamics equations on the plane. SIAM Journal on Mathematical Analysis, Vol. 48, No.1: 443-469.

[8] M. Dai. Local well-posedness of the Hall-MHD system in $H^{s}\left(\mathbb{R}^{n}\right)$ with $s>\frac{n}{2}$. arXiv: 1709.02347, 2017.

[9] M. Dai. Regularity criterion for the 3D Hall-magneto-hydrodynamics. Journal of Differential Equations. Vol. (261): 573-591, 2016.

[10] E. Dumas and F. Sueur. On the weak solutions to the Maxwell-Landau-Lifshitz equations and to the Hall-magnetohydrodynamic equations. Comm. Math. Phys., 330: 1179-1225, 2014.

[11] T. G. Forbes. Magnetic reconnection in solar flares. Geophys. astropphys. fluid dynamics, 62: 15-36, 1991.

[12] L. Grafakos. Modern Fourier analysis. Second edition. Graduate Texts in Mathematics, 250. Springer, New York, 2009.

[13] P. G. Lemarié-Rieusset. Recent developments in the Navier-Stokes problem. Chapman and Hall/CRC Research Notes in Mathematics, 431. Chapman and Hall/CRC, Boca Raton, FL, 2002.

[14] M. J. Lighthill. Studies on magnetohydrodynamic waves and other anisotropic wave motions. Philos. Trans. R. Soc. Lond., Ser. A : 397-430, 1960.

[15] A. J. Majda and A. L. Bertozzi. Vorticity and incompressible flow. Cambridge University Press, 2001.

[16] A. N. Simakov and L. Chacon. Quantitative, analytical model for magnetic reconnection in Hall magnetohydrodynamics. Phys. Rev. Lett, 101, 105003, 2008.

Department of Mathematics, Stat. and Comp.Sci., University of Illinois Chicago, Chichgo, IL 60607,USA

E-mail address: mdai@uic.edu 